\title{
Evaluating the potential costs and impact of digital health technologies for tuberculosis treatment support
}

\author{
Ntwali Placide Nsengiyumva 1,2,3,6, Benjamin Mappin-Kasirer ${ }^{1,2,3,6}$, \\ Olivia Oxlade ${ }^{1,2,3}$, Mayara Bastos ${ }^{4}$, Anete Trajman ${ }^{1,2,3,4}$, Dennis Falzon ${ }^{5}$ and \\ Kevin Schwartzman ${ }^{1,2,3}$
}

Affiliations: ${ }^{1}$ Montreal Chest Institute, Montreal, QC, Canada. ${ }^{2}$ Respiratory Epidemiology and Clinical Research Unit, Centre for Outcomes Research and Evaluation, Research Institute of the McGill University Health Centre, Montreal, QC, Canada. ${ }^{3}$ McGill International Tuberculosis Centre, Montreal, QC, Canada. ${ }^{4}$ State University of Rio de Janeiro, Rio de Janeiro, Brazil. ${ }^{5}$ Global TB Programme, World Health Organization, Geneva, Switzerland. ${ }^{6}$ Both authors contributed equally.

Correspondence: Kevin Schwartzman, McGill University Health Centre, 1001 boulevard Décarie, Room D05.2511, Montreal, QC, H4A 3J1, Canada. E-mail: kevin.schwartzmandmcgill.ca

@ERSpublications

Digital technologies may reduce costs and improve treatment support for persons treated for active TB, and may promote adherence to treatment for latent TB infection in a cost-effective manner http://ow.ly/7MU930lvDWB

Cite this article as: Nsengiyumva NP, Mappin-Kasirer B, Oxlade O, et al. Evaluating the potential costs and impact of digital health technologies for tuberculosis treatment support. Eur Respir J 2018; 52: 1801363 [https://doi.org/10.1183/13993003.01363-2018].

ABSTRACT Ensuring adherence and support during treatment of tuberculosis (TB) is a major public health challenge. Digital health technologies could help improve treatment outcomes. We considered their potential cost and impact on treatment for active or latent TB in Brazil.

Decision analysis models simulated two adult cohorts with 1) drug-susceptible active TB, and 2) multidrug-resistant TB, and two cohorts treated with isoniazid for latent TB infection (LTBI): 1) close contacts of persons with active TB, and 2) others newly diagnosed with LTBI. We evaluated four digital support strategies: two different medication monitors, synchronous video-observed therapy (VOT), and two-way short message service (SMS). Comparators were standard directly observed treatment for active TB and self-administered treatment for LTBI. Projected outcomes included costs (2016 US dollars), plus active TB cases and disability-adjusted life years averted among persons with LTBI.

For individuals with active TB, medication monitors and VOT are projected to lead to substantial (up to $58 \%)$ cost savings, in addition to alleviating inconvenience and cost to patients of supervised treatment visits. For LTBI treatment, SMS and medication monitors are projected to be the most cost-effective interventions. However, all projections are limited by the scarcity of published estimates of clinical effect for the digital technologies.

This article has supplementary material available from erj.ersjournals.com

This is one of a selection of articles published open access, as part of an initiative agreed between the European Respiratory Society and the World Health Organization.

Received: Dec 232017 | Accepted after revision: Aug 192018

Copyright OERS 2018. This version is distributed under the terms of the Creative Commons Attribution NonCommercial Licence 4.0. 


\section{Introduction}

\section{Tuberculosis burden and treatment challenges}

Approximately one quarter of the world's population is estimated to have latent tuberculosis infection (LTBI) [1, 2], and each year, about 10 million individuals develop active tuberculosis (TB) [3, 4]. Despite the success of some strategies to reduce the global TB burden [5], TB remains a leading cause of mortality worldwide, causing an estimated 1.7 million deaths in 2016. Implementation challenges impede the success of directly observed treatment (DOT), as TB treatment is labour intensive for both patients and healthcare providers, requiring rigorous commitment from all to achieve good adherence and treatment success [6]. An effective DOT programme allows early detection and mitigation of suboptimal adherence, which otherwise leads to poorer patient outcomes, including acquired drug resistance [7] and increased costs for both the health system and patient. The latest global treatment success rates reported in 2016 were $83 \%$ among new and relapse TB patients, and 54\% among persons with multidrug-resistant (MDR) TB (resistance to rifampicin and isoniazid) [4].

The World Health Organization (WHO) End TB Strategy aims to halt the TB epidemic by 2035. Beyond better adherence to treatment for active TB, this strategy also calls for improved detection and management of LTBI in countries with high and low TB burdens [8-10]. Obstacles to the uptake and completion of LTBI therapy include the need for testing and medical evaluation, treatment length and risks, and the necessity of follow-up visits with care providers [11, 12]. LTBI treatment is generally self-administered without supervision; consequently, adherence is inconsistent. A meta-analysis published in 2016 estimated that only $62 \%$ of persons starting preventive therapy completed treatment [11]. Only $13 \%$ of eligible contacts aged $<5$ years and $42 \%$ of people newly enrolled in HIV care were estimated to have started preventive treatment for LTBI in 2016, well below the target of $\geqslant 90 \%$ envisaged for these subpopulations by the End TB Strategy for 2025 [4,13]. Measures to improve treatment coverage and completion could increase the contribution of preventive therapy to reduce global TB incidence in the coming years.

\section{Adherence support}

Digital technologies have been evaluated extensively and used successfully to improve outcomes in HIV and other chronic diseases. They have been associated with improved adherence to treatment, with some initial evidence in the TB context [14-17]. The WHO guidelines on the management of LTBI recommend monitoring treatment using digital technologies [8] to further patient-centred care and support, key elements of the TB elimination framework [9]. Indeed, in 2016, WHO's Global Consultation on the Programmatic Management of LTBI called for digital technologies to be used in managing and treating LTBI [18], and in 2017 the Global TB Programme of the WHO released its first evidence-based recommendations on the use of digital technologies in support of TB treatment administration and adherence [19]. These guidelines acknowledged that further evidence is needed to understand the feasibility of implementing digital health technologies, and to measure the impact they could have on TB prevention and care [20-22]. In particular, evidence of the clinical impact of these interventions remains limited to date [17].

Published evaluations of digital health interventions in active TB and LTBI care have provided limited information about cost $[23,24]$. Each digital health technology may entail substantial investment in hardware, software and/or infrastructure. Given the scarce funds for TB prevention and care, such costs must be considered alongside any anticipated gains in health outcomes. In this study, we used decision analysis models to begin to explore the costs and potential clinical impacts of the widely available and scalable technologies that are currently best positioned to replace in-person treatment observation either for active TB and/or to support treatment for LTBI that is currently self-administered. The technologies considered included video-observed therapy (VOT), electronic medication monitors and two-way text messaging (short message service; SMS). We considered their use in Brazil, a high TB burden setting with widespread access to smartphones and telephone network/internet coverage.

\section{Methods}

\section{General description of models}

For active TB disease, a decision analysis model was developed to simulate cohorts of 35-year-old adults (both sexes) who initiate TB treatment in Brazil. Patients were assumed to have either drug-susceptible TB (model 1) or MDR-TB (model 2). The simulation began at treatment initiation and allowed for two rounds of treatment if needed, representing a time horizon of 2-4 years. Two digital health interventions, VOT and medication monitors, were compared against standard DOT for treatment support of drug-susceptible TB and MDR-TB. We did not consider SMS support of active TB treatment, for reasons detailed later. 
For LTBI, a decision analysis model simulated two cohorts of 35-year-old adults (both sexes) diagnosed with LTBI in Brazil. One cohort was comprised of individuals diagnosed with LTBI who were close contacts of drug-sensitive active TB cases. The other cohort consisted of members of the general population diagnosed with LTBI. Simulations began at treatment initiation and lasted 20 years. 9 months of daily isoniazid, supported by SMS, VOT or medication monitors, were compared with self-administered treatment (SAT), the standard of care for LTBI.

In all models, outcomes included disability-adjusted life years (DALYs) and costs for each strategy. For the LTBI cohorts, future incident TB cases were also projected.

Costs were expressed in 2016 US dollars (USD) and were projected from both the health system and societal perspectives. Where appropriate, cost-effectiveness was evaluated using incremental cost-effectiveness ratios. Models were developed using TreeAge Pro 2016 software (TreeAge Software Inc., Williamstown, MA, USA). Simplified schemas of the decision analysis models are included in the supplementary material.

\section{Study setting}

Brazil is a high TB burden country: in 2016 it had an estimated incidence of 87000 TB cases (95\% CI 74000-100 000) at a rate of 42 cases per 100000 population [4]. It has a comprehensive disease database, the Notification of Injury Information System (Sistema de Informação de Agravos de Notificação (SINAN); http://portalsinan.saude.gov.br), providing up-to-date clinical and TB programme data. This setting was chosen in part because of Brazil's high mobile-broadband penetration; in 2015 it had an $88 \%$ penetration, and was ranked fourth globally in active internet users [25].

\section{Digital interventions}

Digital health interventions were selected on the basis of a recent systematic review of digital technologies for TB [17]. The following sections give summaries of the strategies included. Table 1 provides more operational details and summarises the effect estimates for all digital interventions considered.

\section{Medication monitors}

Two medication monitor strategies were considered for treatment support for both active and latent TB: Wisepill (Wisepill Technologies, Somerset West, South Africa) and 99DOTS (an open-source toolkit). A randomised trial of treatment support for active TB in China reported that patients using medication monitors had 0.58 (95\% CI $0.42-0.79$ ) times the number of patient-months with $20 \%$ or more missed doses as those on standard care [33]. However, an impact on clinical outcomes was not demonstrated. In the primary analysis, we therefore made the conservative assumption that for active $\mathrm{TB}$, medication monitors would yield treatment outcomes comparable to standard care with direct observation. There are no randomised studies evaluating medication monitors in LTBI care. Thus, in the LTBI model, we assumed that medication monitors would lead to better adherence than SAT in a context where support for LTBI treatment is very limited. We derived estimates of effect from the same trial in China [33]. A relative risk of 1.18 (95\% CI 1.08-1.26) for treatment completion was calculated using published data from the Chinese trial (see supplementary material for detailed calculations).

Video-observed therapy

VOT was considered for treatment support for both active and latent TB. The VOT intervention was adapted from an observational study of active TB treatment support in New York City (USA) in 2014 [31]. The New York City study reported adherence with VOT of $95 \%$ of doses, compared to $91 \%$ for in-person DOT $(\mathrm{p}<0.01)$. In our active TB model, we thus assumed that treatment success rates using VOT for active TB were equivalent to those for in-person DOT. There are no published trials or other studies of VOT performed to monitor LTBI treatment. We assumed that VOT was likely to improve adherence to LTBI treatment relative to SAT. Thus, we assumed that the effect of VOT for LTBI would be equivalent to that of medication monitors.

\section{SMS}

Because randomised studies have suggested that SMS is not well suited to active TB care, we did not model this intervention in the context of active TB [35]. The two-way SMS intervention for LTBI was adapted from a study from British Columbia, Canada [32]. LTBI is usually treated using SAT, as is the case for HIV. We relied on the largest published randomised trial of two-way SMS for HIV treatment in Kenya (538 participants) [34], and assumed that the effect of two-way SMS on treatment completion was similar for LTBI and HIV. Again, extrapolating from the Kenyan trial, we assumed that SMS was likely to improve treatment support and completion in a context where existing support for LTBI treatment is very limited. 
TABLE 1 Details of digital support interventions for treatment of active tuberculosis (TB) and latent TB infection (LTBI)

$\begin{array}{lll}\begin{array}{l}\text { Digital } \\ \text { technologies }\end{array} & \text { Operational details } & \text { Estimate of effect }\end{array}$

technologies

Active TB

LTB

MM: Wisepill Small device attached to standard pill dispenser

When opened, device communicates with web-based application by SMS

Device sends SMS to patient and HCW when daily dose is missed

One-way texting: no response is required of patient [26-28]

MM: 99DOTS

Taking pills from blister pack daily reveals random toll-free numbers

Any call from a registered patient number is marked as a dose taken

When patients call, they hear "thank you"

Automatic alerts to patients and HCW of missed doses

VOT

pen-source information technology system $[29,30]$

Smartphone loaned to patients without one

Pre-arranged schedule for real-time (synchronous) daily VOT calls with HCW

Patient shows and names pills, then swallows them

Patient asked about adverse reactions

Missed appointments followed up first by phone calls, then by home visits [31]

Two-way SMS Weekly SMS “check-ins" sent from central computer at clinic

Patients asked to respond within $24 \mathrm{~h}$

First instance of non-response: follow-up SMS

Second instance of non-response: phone call [32]
Assumed effect was

equivalent to DOT/standard of care

Assumed effect was equivalent to DOT/standard of care

Assumed effect was equivalent to DOT/standard

Not estimated

$$
\text { of care }
$$

Assumed effect was equivalent to that of MM in trials of active TB
Assumed effect was equivalent to that of MM in trials of active TB

Assumed effect was equivalent to that of MM in trials of active TB

MM: medication monitor; VOT: video-observed therapy; SMS: short message service; HCW: healthcare worker; DOT: directly observed treatment. " : routine in-person DOT visits were replaced by digital technologies for active TB treatment supervision; १: patients using digital interventions were $1.18(95 \% \mathrm{Cl} 1.08-1.26)$ times more likely to complete treatment than those on self-administered treatment (derived from [33]); ${ }^{+}$: patients using digital intervention were 1.24 (95\% Cl 1.06-1.45) times more likely to complete treatment than those on self-administered treatment [34].

\section{Data sources}

Pathogenesis, natural history of TB, epidemiology and drug resistance data were taken from published literature, and are summarised in supplementary table S3. Treatment outcomes were derived from 20132014 country-specific data from the Brazilian national TB programme (supplementary table S1). For cost-utility analyses, a weight of 0.331 DALYs was attributed to active TB (95\% CI 0.224-0.454) [36], and a weight of 0 DALYs was attributed to LTBI, as individuals with LTBI are asymptomatic (supplementary table S3). Further description of the DALY calculations is provided in the supplementary material.

Costs

All costs were expressed in 2016 USD, using relevant exchange rates and published inflation indices for Brazil and for any other countries from which cost data were obtained (see supplementary material). Costs obtained from India and South Africa were normalised to the Brazilian per capita gross national income (GNI), by multiplying them by the number resulting from dividing Brazilian per capita GNI by Indian or South African per capita GNI for the relevant year. During the 20-year simulation of individuals with LTBI, all future costs and outcomes were discounted at an annual rate of $3 \%$.

\section{Digital technology costs}

Technology costs included materials required to implement the interventions, such as phones, dispensers and envelopes. Start-up and follow-on operational costs for each technology, training costs and staff pay based on the time needed to complete an activity, including steps taken to follow up with patients reporting problems or demonstrating suboptimal adherence, were also incorporated. Table 2 serves as an example of the types of cost components included for the SMS intervention and is shown as a per person cost. Detailed cost breakdowns for all other interventions are shown in supplementary table S9. 
Treatment-related health system costs

Health system costs included staff salaries for all DOT and other follow-up visits, costs of follow-up monitoring tests and drug costs. DOT visits were assumed to occur three times weekly, as recommended by Brazilian authorities [41], with other treatment doses self-administered. For re-treatment, diagnostic costs were also included. Hospitalisation and adverse event management costs were based on published estimates [42, 43] (supplementary tables S4-S7). Patient costs were classified as direct (out-of-pocket expenses related to medical visits) and indirect (time lost due to medical visits) (supplementary table S8). Table 3 summarises aggregate cost inputs used for standard treatment regimens for the different cohorts and for each of the intervention scenarios. These aggregate costs reflect the costs incurred by the health system and patient for diagnosis and treatment (varying depending on type of TB), plus any additional costs relevant to each of the specific digital technologies (as shown for SMS in table 2).

\section{Scenario and sensitivity analyses}

Due to the paucity of empirical data on clinical outcomes with the digital interventions, we considered several alternative scenarios to those already described. For active TB, we considered the possibility that medication monitors and VOT may actually improve TB treatment outcomes relative to DOT. In this scenario, we assumed that improved adherence as reported in China would lead to improved treatment success rates, and applied the ratio reported by Liu et al. [33] to unfavourable treatment outcomes (loss to follow-up, death and failure) in the medication monitor and VOT intervention arms of the model.

An alternative analysis for the LTBI cohorts focused only on cost differences between scenarios, setting the effect of all interventions to be equivalent to that used for medication monitors in the baseline analyses (i.e. relative risk of 1.18 for treatment completion, compared to SAT). Additionally, a threshold analysis was conducted for the LTBI cohort to identify the minimal efficacy required of the intervention in order for it to remain cost-effective when considering DALYs averted.

Probabilistic sensitivity analyses were used to generate 95\% uncertainty ranges (UR; 2.5th to 97.5th percentiles) around point estimates for all projected outcomes. They were also used to further explore the potential cost-effectiveness of these technologies. Additional sensitivity analyses, including one-way

TABLE 2 Digital technology costs for treatment support using two-way short message service (SMS), per person with latent tuberculosis infection (LTBI), in 2016 USD $^{\#}$

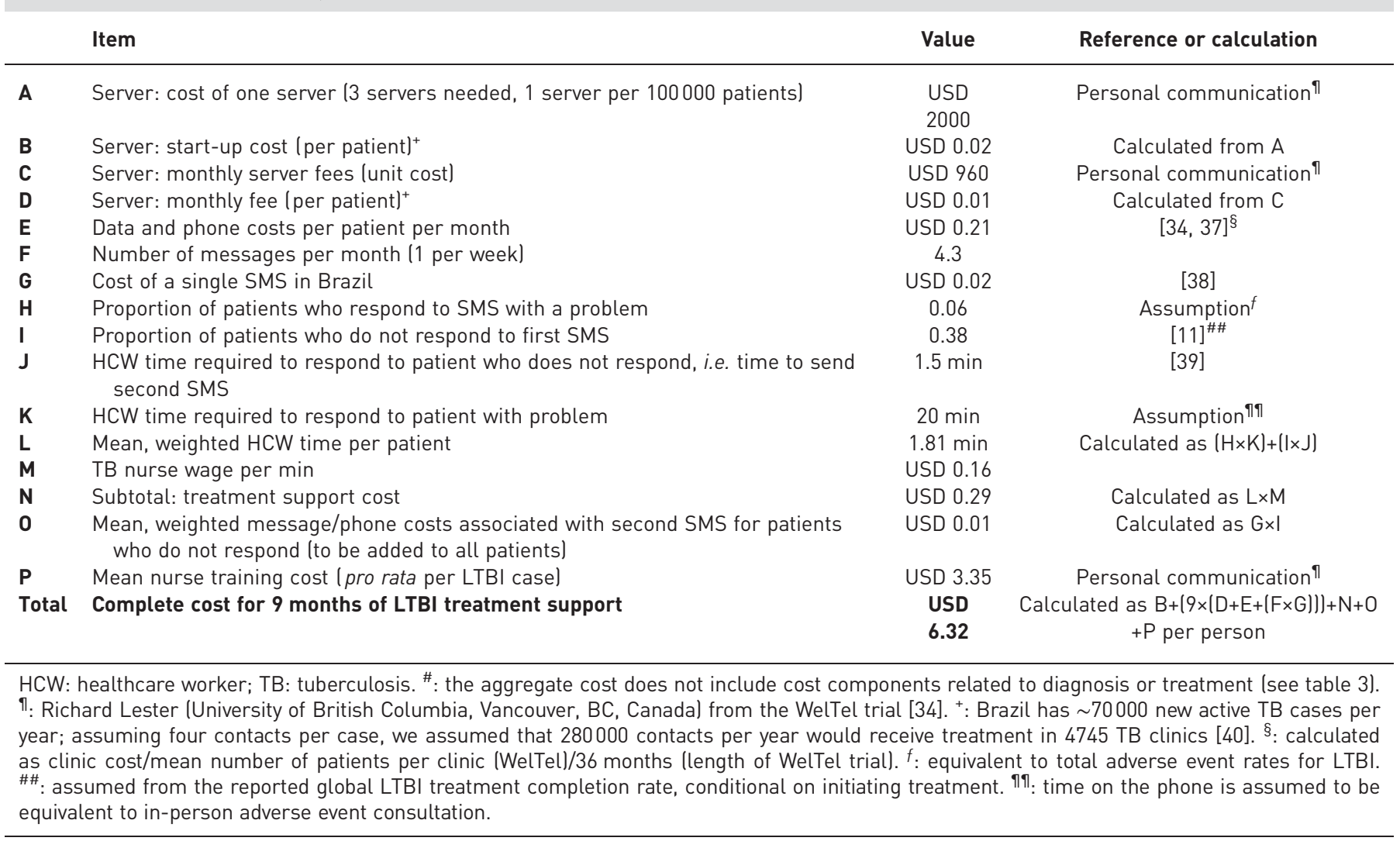


TABLE 3 Total input cost per person with tuberculosis (TB) or latent TB infection (LTBI) for diagnosis and treatment, by type of digital technology used for treatment support, in 2016 USD

\begin{tabular}{|c|c|c|c|c|c|c|c|c|}
\hline & \multirow{2}{*}{$\begin{array}{l}\text { Treatment } \\
\text { length } \\
\text { months }\end{array}$} & \multicolumn{5}{|c|}{ Total costs incurred by health system } & \multicolumn{2}{|c|}{$\begin{array}{c}\text { Total costs incurred by } \\
\text { patient }\end{array}$} \\
\hline & & $\begin{array}{l}\text { Standard } \\
\text { of care }\end{array}$ & VOT & $\begin{array}{c}\text { MM: } \\
\text { Wisepill }\end{array}$ & $\begin{array}{c}\text { MM: } \\
\text { 99DOTS }\end{array}$ & SMS & $\begin{array}{l}\text { Standard } \\
\text { of care }\end{array}$ & $\begin{array}{c}\text { Digital } \\
\text { technology }\end{array}$ \\
\hline Drug-susceptible ТВ & 6 & 845 & 505 & 368 & 340 & NA & 299 & 140 \\
\hline MDR-TB & 18 & 10014 & 8879 & 8527 & 8494 & NA & 813 & 337 \\
\hline LTBI & 9 & 53 & 397 & 91 & 61 & 59 & 148 & 148 \\
\hline
\end{tabular}

Costs listed account for pre-diagnostic management, diagnosis, treatment and follow-up. VOT: video-observed therapy; MM: medication monitor; SMS: short message service; MDR: multidrug-resistant; NA: not applicable.

sensitivity analyses for key parameters used in each model and relevant tornado diagrams, are described in the supplementary material.

\section{Results}

\section{Health system perspective}

Drug-susceptible TB and MDR-TB cohorts

Assuming equivalent drug-susceptible TB treatment success rates with DOT and digital interventions, all interventions led to health systems cost savings relative to standard DOT, ranging from 39\% to 58\%. For the MDR-TB cohort, all digital interventions also led to health system cost savings, where treatment success rates were assumed to be equivalent to those with DOT. Relative to DOT, cost savings ranged from $11 \%$ to $15 \%$. Further details are provided in table 4.

\section{LTBI cohorts}

Among close contacts with LTBI, SMS and the medication monitors were projected to be most cost-effective compared to SAT. The incremental cost of SMS was USD 5 per person, with a reduction in DALYs of 0.04 per person, and a $0.8 \%$ absolute decrease in future TB incidence over the 20-year simulation. The incremental cost of SMS was USD 123 per DALY averted, and USD 611 per TB case prevented. Both medication monitors and VOT averted 0.03 DALYs per person, and led to an absolute reduction in TB incidence of $0.6 \%$. The 99DOTS medication monitor was the second most cost-effective intervention, with an incremental cost of USD 210 per DALY averted, and USD 1038 per TB case prevented. The Wisepill medication monitor had an incremental cost of USD 1178 per DALY averted, and USD 5836 per TB case prevented. VOT had an incremental cost of USD 9805 per DALY averted, and USD 48551 per TB case prevented. Complete results are shown in table 5.

Among persons from the general population with LTBI, SMS was the most cost-effective intervention. SMS cost USD 1000 per DALY averted and USD 4483 per TB case prevented compared to SAT (table 5).

\section{Societal perspective}

Drug-susceptible TB and MDR-TB cohorts

Combining health system and patient costs, cost savings with digital technologies for the drug-susceptible TB cohort ranged from $43 \%$ to $56 \%$, and from $15 \%$ to $18 \%$ for MDR-TB, relative to standard DOT. Estimated savings to patients were USD 168 for each drug-susceptible TB patient and USD 593 for each MDR patient. See table 4 for full details.

\section{LTBI cohorts}

In both LTBI cohorts, results from the societal perspective (table 5) were similar to those from the health system perspective. In close contacts, SMS was projected to be the most cost-effective and VOT the least. As expected, all interventions were less cost-effective in unselected persons with LTBI. The highest incremental cost-effectiveness ratios were for VOT, with estimated costs of USD 44042 per DALY averted and USD 197411 per TB case prevented.

\section{Scenario and sensitivity analyses}

In the additional analysis for the active TB cohort, where medication monitors and VOT were assumed to lead to better treatment outcomes than DOT, digital technologies were estimated to improve treatment success rates in the drug-susceptible TB cohort from 71\% with DOT (95\% UR 71-72\%) to 83\% (95\% UR 79-87\%), and to reduce DALYs accrued by 39\%, from 3.2 (95\% UR 3.1-3.3) to 1.9 (95\% UR 1.6-2.4) per 


\begin{tabular}{|c|c|c|}
\hline Digital support strategy & Cost per person & Incremental savings versu \\
\hline \multicolumn{3}{|c|}{ Drug-susceptible TB cohort } \\
\hline \multicolumn{3}{|c|}{ Health system perspective } \\
\hline DOT & 930 (876-1095) & Comparator \\
\hline VOT & $567(529-660)$ & $363(302-490)$ \\
\hline MM: Wisepill & $423(392-511)$ & $507(450-631)$ \\
\hline MM: 99DOTS & $394(363-482)$ & $536(479-660)$ \\
\hline \multicolumn{3}{|l|}{ Societal perspective } \\
\hline DOT & $1249(903-3069)$ & Comparator \\
\hline VOT & $718(572-1170)$ & $531(69-2320)$ \\
\hline MM: Wisepill & $574(431-1032)$ & $675(217-2394)$ \\
\hline MM: 99DOTS & 545 (402-1003) & 704 (249-2463) \\
\hline \multicolumn{3}{|l|}{ MDR-TB cohort } \\
\hline \multicolumn{3}{|c|}{ Health system perspective } \\
\hline DOT & 12585 (12395-13184) & Comparator \\
\hline VOT & 11177 (11027-11563) & $1409(1212-1826)$ \\
\hline MM: Wisepill & 10754 (10623-11 128) & $1831(1648-2235)$ \\
\hline MM: 99DOTS & 10715 (10584-11089) & $1870(1686-2274)$ \\
\hline \multicolumn{3}{|l|}{ Societal perspective } \\
\hline DOT & 13650 (12537-19923) & Comparator \\
\hline VOT & 11648 (11224-12769) & $2002(371-8095)$ \\
\hline MM: Wisepill & 11225 (10817-12358) & 2425 (789-8195) \\
\hline MM: 99DOTS & $11186(10779-12319)$ & $2463(828-8234)$ \\
\hline
\end{tabular}

Data are presented as cost or savings with $95 \%$ uncertainty ranges. DOT: directly observed treatment; VOT: video-observed therapy; MM: medication monitor; MDR: multidrug-resistant.

patient. With this scenario, societal cost savings relative to DOT were USD 543 with VOT, USD 687 with the Wisepill medication monitor and USD 716 with the 99DOTS medication monitor. For the MDR-TB cohort, these technologies were projected to improve treatment success rates from 59\% with standard DOT (95\% UR 57-62\%) to 75\% (95\% UR 69-80\%), and reduce DALYs by 35\%, from 5.2 (95\% UR 4.8-5.6) to 3.4 (95\% UR 2.9-4.2) per patient.

The additional scenario analysis for our LTBI model, which set effectiveness equal for all digital interventions so as to compare their costs, yielded results consistent with our primary findings. In this analysis, two-way SMS and 99DOTS medication monitor remained the most cost-effective interventions: the incremental cost (95\% UR) of SMS was USD 164 (USD 29 saving to USD 362 cost) per DALY averted, and USD 814 (USD 137 saving to USD 1781 cost) per TB case prevented.

In the active TB cohorts, the probabilistic sensitivity analysis suggested that medication monitors and VOT were likely to be cost saving within all model parameter ranges considered. For the LTBI models, SMS, 99DOTS medication monitor and Wisepill medication monitor were cost-effective (using USD 8650, the mean per capita gross domestic product in Brazil, as the willingness-to-pay threshold) relative to SAT in $100 \%$ of simulations among close contacts of persons with LTBI. Among members of the general population newly diagnosed with LTBI, these technologies were cost-effective in more than $90 \%$ of simulations, using the same willingness-to-pay threshold. VOT was cost-effective in only $60 \%$ of simulations in the cohort of close contacts, and was not cost-effective among members of the general population with LTBI.

\section{Discussion}

This analysis is the first to examine the potential cost and impact of digital technologies as applied under programme conditions in a high TB burden country. It provides preliminary insights into the potential impact and cost of several approaches to TB treatment support in this context. For active TB, we estimated substantial cost savings with VOT and medication monitors, including savings to patients and their families, compared to conventional in-person DOT. Compared to SAT for LTBI treatment, two-way SMS and medication monitors are projected to be the most cost-effective technologies, especially for close contacts with LTBI; they are less cost-effective for others with LTBI. Until VOT becomes cheaper, it will probably be substantially less cost-effective for supporting LTBI treatment as currently delivered in Brazil and similar settings, despite improvements in rates of treatment completion. Although Brazil was the focus 
TABLE 5 Projected cost-effectiveness of strategies using digital support for latent tuberculosis infection (LTBI) treatment, in 2016 USD

\begin{tabular}{|c|c|c|c|c|c|c|}
\hline Digital support strategy & $\begin{array}{l}\text { Cost per } \\
\text { person }\end{array}$ & $\begin{array}{l}\text { Incremental cost } \\
\text { versus SAT }\end{array}$ & DALYs accrued & $\begin{array}{l}\text { Incremental cost per DALY } \\
\text { averted versus SAT" }\end{array}$ & $\begin{array}{c}\text { TB incidence } \\
\%\end{array}$ & $\begin{array}{l}\text { Incremental cost per TB case } \\
\text { prevented versus SAT }\end{array}$ \\
\hline \multicolumn{7}{|l|}{ LTBI cohort: close contacts } \\
\hline \multicolumn{7}{|c|}{ Health system perspective } \\
\hline SAT & $60(50-103)$ & & $0.1(0.07-0.24)$ & & $2.0(1.5-4.6)$ & \\
\hline MM: Wisepill & $96(84-136)$ & $36(29-38)$ & $0.07(0.05-0.17)$ & $1178(375-2171)$ & $1.4(1.1-3.4)$ & 5836 (1875-10 704) \\
\hline MM: 99DOTS & $67(55-107)$ & $7(-7-8)$ & $0.07(0.05-0.17)$ & $210(-10-445)$ & $1.4(1.1-3.4)$ & $1038(-40-2195)$ \\
\hline VOT & $356(314-421)$ & $296(246-340)$ & $0.07(0.05-0.17)$ & 9805 (3658-17596) & $1.4(1.1-3.4)$ & 48551 (18744-83722) \\
\hline SMS & 65 (53-105) & $5(-6-8)$ & $0.06(0.03-0.16)$ & $123(-57-311)$ & $1.2(0.8-3.1)$ & $611(-273-1556)$ \\
\hline \multicolumn{7}{|l|}{ Societal perspective } \\
\hline SAT & 181 (79-477) & & $0.1(0.07-0.24)$ & & $2.0(1.5-4.6)$ & \\
\hline MM: Wisepill & $226(114-546)$ & $45(24-71)$ & $0.07(0.05-0.17)$ & 1495 (389-2801) & $1.4(1.1-3.4)$ & 7404 (1816-13477) \\
\hline MM: 99D0TS & $197(85-517)$ & $16(-5-42)$ & $0.07(0.05-0.17)$ & $527(-100-1356)$ & $1.4(1.1-3.4)$ & $2608(-564-6414)$ \\
\hline VOT & $486(358-806)$ & $305(250-355)$ & $0.07(0.05-0.17)$ & $10122(3730-17547)$ & $1.4(1.1-3.4)$ & 50119 (18756-86092) \\
\hline SMS & $198(82-531)$ & $17(-13-58)$ & $0.06(0.03-0.16)$ & $440(-168-1223)$ & $1.2(0.8-3.1)$ & $2180(-819-5837)$ \\
\hline \multicolumn{7}{|c|}{$\begin{array}{l}\text { LTBI cohort: general population } \\
\text { newly diagnosed with LTBI } \\
\text { Health system perspective }\end{array}$} \\
\hline SAT & 50 (39-83) & & $0.030(0.030-0.055)$ & & $0.7(0.6-1.2)$ & \\
\hline MM: Wisepill & $89(76-124)$ & $39(37-41)$ & $0.023(0.022-0.044)$ & 5520 (2722-7818) & $0.5(0.5-1.0)$ & 24745 (12327-35 197) \\
\hline MM: 99D0TS & $60(46-94)$ & $10(8-12)$ & $0.023(0.022-0.044)$ & $1370(639-1849)$ & $0.5(0.5-1.0)$ & $6180(2893-8273)$ \\
\hline VOT & $349(303-412)$ & $299(251-346)$ & $0.023(0.022-0.044)$ & $42481(20534-60702)$ & $0.5(0.5-1.0)$ & 190415 (92588-271118) \\
\hline SMS & $59(45-95)$ & $9(7-13)$ & $0.021(0.017-0.042)$ & $1000(383-1479)$ & $0.5(0.3-0.9)$ & $4483(1755-6632)$ \\
\hline \multicolumn{7}{|l|}{ Societal perspective } \\
\hline SAT & 167 (63-445) & & $0.030(0.030-0.055)$ & & $0.7(0.6-1.2)$ & \\
\hline MM: Wisepill & $216(101-519)$ & $49(38-76)$ & $0.023(0.022-0.044)$ & 7081 (3080-10460) & $0.5(0.5-1.0)$ & 31740 (14115-45303) \\
\hline MM: 99DOTS & $187(72-490)$ & $20(8-47)$ & $0.023(0.022-0.044)$ & 2931 (804-5508) & $0.5(0.5-1.0)$ & $13136(3656-24628)$ \\
\hline VOT & $477(342-783)$ & $310(256-362)$ & $0.023(0.022-0.044)$ & $44042(20986-62452)$ & $0.5(0.5-1.0)$ & 197411 (96567-278873) \\
\hline SMS & $190(72-507)$ & $23(7-68)$ & $0.021(0.017-0.042)$ & $2561(535-5166)$ & $0.5(0.3-0.9)$ & 11479 (2407-22869) \\
\hline
\end{tabular}


of our analysis, these results could potentially be relevant to other settings (recognising that treatment practices will vary, e.g. in many settings, 6 months of isoniazid is the norm when LTBI is treated).

Our analysis highlights the paucity of published data on the clinical impact of digital interventions for TB. Few randomised trials exist and many of the published results are from studies in very specific treatment settings, e.g. for active TB treatment in New York and China and HIV treatment in Kenya; these may not be generalisable to other populations and clinical settings including active TB and LTBI treatment in Brazil. Consequently, our analyses relied on estimates of clinical effectiveness that were often extrapolated from short-term data regarding adherence. Moreover, we based several estimates on the Chinese study of medication monitors where the control arm involved a mix of DOT and SAT, which could overestimate the benefit of medication monitors when compared with true DOT. We investigated the impact of uncertainty in our parameter estimates through extensive sensitivity analyses. However, if subsequent field trials suggest poorer clinical outcomes for a given digital support technology versus conventional DOT, that technology is unlikely to be implemented further in active TB treatment, regardless of associated cost savings.

Published data on the costs of digital interventions are also scarce and sometimes out-dated. Particularly in low- and middle-income countries, technology-related costs can change rapidly due to improvements in infrastructure, such as internet connectivity. Whenever possible, we contacted study authors and manufacturers to obtain the best available cost estimates, and accounted for variability and uncertainty in sensitivity analyses. It should be noted that technology costs considered in this study do not include initial technology development and upstream support as they are beyond the scope of our analyses. There are also costs inherent to implementing technologies at large scale (beyond costs of training, hardware and software), which are difficult to capture.

In our study, we assumed that costs and outcomes for standard care of active TB reflected direct observation. This assumption almost certainly overestimated the total number and cost of DOT visits that in fact occur under programme conditions. We did not have data for the precise number of DOT visits that actually take place. We may also have overestimated use of Xpert MTB/RIF (Cepheid, Sunnyvale, CA, USA) for TB diagnosis, which could lead to some overestimation of diagnostic costs for persons with LTBI who subsequently develop active TB. Furthermore, our models assumed 100\% adoption of the digital technologies. Under programme conditions, uptake is likely to be less, a point again explored via varying efficacy estimates in sensitivity analysis. Additional analyses involving variable uptake as well as combinations of these technologies, or only partial replacement of DOT in active TB, could provide further insight.

In Brazil, the estimated costs of TB for the health system and for patients and families are substantially lower than in a higher-income setting like the USA. For example, CASTRO et al. [44] estimated health system costs of USD 17000 for each case of drug-susceptible TB, plus additional costs to patients and families of USD 3000 and lost income of USD 374000 for each patient who dies prematurely from drug-susceptible TB. Hence, to the extent that digital technologies reduce health system costs, and might potentially improve treatment outcomes in LTBI, resulting savings could be greater in higher-income settings. In the case of LTBI, where we assumed improved treatment adherence with the digital technologies, and hence a lower risk of future reactivation, we did not consider societal cost savings related to reductions in subsequent death and long-term disability. However, we did estimate DALYs averted.

In the future, digital support strategies may not only serve to improve adherence to TB and LTBI treatment, but may also improve monitoring of adverse events, a particular concern with SAT. SMS and VOT could allow patients to report potential adverse reactions and other concerns in a more timely manner, potentially improving outcomes and reducing costs. Our models could not account for this additional advantage, due to the lack of published evidence describing it. As additional trial data become available, this will be a worthwhile avenue of inquiry. More generally, digital technologies offer the possibility of detecting adherence gaps, in a less intrusive and expensive manner than traditional DOT. Any such gaps must then be addressed by tailored, patient-centred approaches, which could include digital and/or in-person interactions.

As in other health domains, digital technologies can potentially replace or enhance existing treatment models $[45,46]$, but such a paradigm shift comes with challenges in development, design, implementation, maintenance, accessibility and acceptability [47, 48]. For example, patients theoretically could "cheat" medication monitor systems by opening dispensers or blister packs without ingesting medication; this would falsely be recorded as good adherence $[26,33]$. Although not completely foolproof, VOT maintains direct observation of medication ingestion, but may be perceived as intrusive by some patients [49]. When adopting digital technologies in the TB context, we can draw lessons from other fields where solutions were tailored to the perspectives of patients and providers to enhance feasibility [50, 51]. 
Digital support technologies may be more cost-effective with intermittent isoniazid treatment, or with newer and shorter LTBI medication regimens, such as the 12-dose weekly isoniazid/rifapentine combination. This was initially administered under direct, in-person observation [52]; however, a recent study reported no benefit for one-way SMS reminders added to standard SAT for this regimen [53]. Increasing global availability of these regimens justifies further evaluation of the cost-effectiveness of two-way SMS, medication monitors and VOT to support shorter-course LTBI treatment. It is also conceivable that with higher adherence to the self-administered shorter-course regimens, digital technologies could in fact provide less benefit in this regard; this possibility also warrants further evaluation.

\section{Conclusion}

Although DOT has long been held up as the paragon for active TB treatment support, it is not always possible to implement optimally. Our analysis shows that digital technologies may reduce costs and improve treatment support for persons treated for active TB. However, more evidence of their value is needed, especially in the context of TB care. Furthermore, this analysis again emphasises that LTBI treatment and treatment support should focus on persons at higher risk of developing active TB. By helping to improve the management of LTBI, digital technologies could contribute to reducing TB incidence and meeting the goals of the WHO End TB Strategy.

Acknowledgements: The authors acknowledge the contributions of the members of the Collaborative Group on the Impact of Digital Technologies on Tuberculosis: Ibrahim Abubakar (University College, London, UK); Narges Alipanah (University of California, San Francisco, CA, USA); Delia Boccia (London School of Hygiene and Tropical Medicine, London, UK); Theodore Cohen (Yale University, New Haven, CT, USA); J. Lucian Davis (Yale University); Claudia Denkinger (Foundation for Innovative New Diagnostics (FIND), Geneva, Switzerland); Katherine Fielding (London School of Hygiene and Tropical Medicine); Gregory J. Fox (University of Sydney, Sydney, Australia); Caroline Free (London School of Hygiene and Tropical Medicine); Richard S. Garfein (University of California, San Diego, CA, USA); Andrew Hayward (University College, London); Ernesto Jaramillo (Global TB Programme, World Health Organization, Geneva, Switzerland); Richard Lester (University of British Columbia, Vancouver, BC, Canada); James Lewis (London School of Hygiene and Tropical Medicine); Florian Marx (Yale University); Giovanni Battista Migliori (World Health Organization Collaborating Centre for TB and Lung Diseases and ERS TB Collaborating Centre, Maugeri Care and Research Institute, Tradate, Italy); Payam Nahid (University of California, San Francisco); Kamran Siddiqi (University of York, York, UK); Alistair Story (University College, London); Mohammed Yassin (Global Fund To Fight AIDS, Tuberculosis and Malaria, Geneva, Switzerland).

Conflict of interest: N.P. Nsengiyumva reports grants from the European Respiratory Society, during the conduct of the study. B. Mappin-Kasirer has nothing to disclose. O. Oxlade has nothing to disclose. M. Bastos has nothing to disclose. A. Trajman has nothing to disclose. D. Falzon reports a grant from the European Respiratory Society for activities in support of the digital health agenda for the End TB Strategy (funder not involved in decisions made on the content of the current manuscript), during the conduct of the study. K. Schwartzman reports grants from the European Respiratory Society through the Global TB Programme of the World Health Organization, which paid for some research expenses associated with this work, during the conduct of the study.

Support statement: This study was funded by the European Respiratory Society, through a grant to the World Health Organization Global Tuberculosis Programme. Funding information for this article has been deposited with the Crossref Funder Registry.

\section{References}

1 Getahun H, Matteelli A, Chaisson RE, et al. Latent Mycobacterium tuberculosis infection. N Engl J Med 2015; 372: 2127-2135.

2 Houben RM, Dodd PJ. The global burden of latent tuberculosis infection: a re-estimation using mathematical modelling. PLoS Med 2016; 13: e1002152.

3 Stop TB Partnership, United Nations Office for Project Services. Global Plan to End TB: The Paradigm Shift, 2016-2020. Geneva, Stop TB Partnership, 2015. www.stoptb.org/assets/documents/global/plan/globalplantoendtb_ theparadigmshift_2016-2020_stoptbpartnership.pdf

4 World Health Organization. Global Tuberculosis Report 2017. WHO/HTM/TB/2017.23. Geneva, World Health Organization, 2017. Available from: www.who.int/tb/publications/global_report/en/

5 World Health Organization. TB: A Global Emergency. WHO Report on the Tuberculosis Epidemic. WHO/TB/ 94.177. Geneva, World Health Organization, 1994. Available from: www.who.int/tb/publications/1994/en/

6 Munro SA, Lewin SA, Smith HJ, et al. Patient adherence to tuberculosis treatment: a systematic review of qualitative research. PLoS Med 2007; 4: e238.

7 World Health Organization. Companion Handbook to the WHO Guidelines for the Programmatic Management of Drug-Resistant Tuberculosis. WHO/HTM/TB/2014.11. Geneva, World Health Organization, 2014. Available from: www.who.int/tb/publications/pmdt_companionhandbook/en/

8 Getahun H, Matteelli A, Abubakar I, et al. Management of latent Mycobacterium tuberculosis infection: WHO guidelines for low tuberculosis burden countries. Eur Respir J 2015; 46: 1563-1576.

9 Lönnroth K, Migliori GB, Abubakar I, et al. Towards tuberculosis elimination: an action framework for low-incidence countries. Eur Respir J 2015; 45: 928-952. 
10 Patel AR, Campbell JR, Sadatsafavi M, et al. Burden of non-adherence to latent tuberculosis infection drug therapy and the potential cost-effectiveness of adherence interventions in Canada: a simulation study. BMJ Open 2017; 7: e015108.

11 Alsdurf H, Hill PC, Matteelli A, et al. The cascade of care in diagnosis and treatment of latent tuberculosis infection: a systematic review and meta-analysis. Lancet Infect Dis 2016; 16: 1269-1278.

12 Fiske CT, Yan FX, Hirsch-Moverman Y, et al. Risk factors for treatment default in close contacts with latent tuberculous infection. Int J Tuberc Lung Dis 2014; 18: 421-427.

13 World Health Organization. Implementing the End TB Strategy: The Essentials. WHO/HTM/TB/2015.31. Geneva, World Health Organization, 2015. Available from: www.who.int/tb/publications/2015/The_Essentials_to_End_TB/en/

14 Marcum ZA, Gellad WF. Improving medication adherence: keep your eyes on the prize. J Gen Intern Med 2017; 32: 236-237.

15 Zullig LL, Shaw RJ, Bosworth HB. Applying technology to medication management and adherence. In: Marsch L, Lord S, Dallery J, eds. Behavioral Health Care and Technology: Using Science-Based Innovations to Transform Practice. Oxford, Oxford University Press, 2014; pp. 81-94.

16 Reddy A, Huseman TL, Canamucio A, et al. Patient and partner feedback reports to improve statin medication adherence: a randomized control trial. J Gen Intern Med 2017; 32: 256-261.

17 Ngwatu BK, Nsengiyumva NP, Oxlade O, et al. The impact of digital health technologies on tuberculosis treatment: a systematic review. Eur Respir J 2018; 51: 1701596.

18 World Health Organization. Report of the Global Consultation on the Programmatic Management of Latent Tuberculosis Infection. WHO/HTM/TB/2016.08. Geneva, World Health Organization, 2016. Available from: www. who.int/tb/publications/korea_consultation_report_apr2016/en/

19 World Health Organization. Guidelines for the Treatment of Drug-Susceptible Tuberculosis and Patient Care, 2017 update. WHO/HTM/TB/2017.05. Geneva, World Health Organization, 2017. Available from: www.who.int/ tb/publications/2017/dstb_guidance_2017/en/

20 Falzon D, Timimi H, Kurosinski P, et al. Digital health for the End TB Strategy: developing priority products and making them work. Eur Respir J 2016; 48: 29-45.

21 Story A, Garfein RS, Hayward A, et al. Monitoring therapy compliance of tuberculosis patients by using video-enabled electronic devices. Emerg Infect Dis 2016; 22: 538-540.

22 World Health Organization. Digital Health for the End TB Strategy: An Agenda for Action. WHO/HTM/TB/ 2015.21. Geneva, World Health Organization, 2015. Available from: www.who.int/tb/publications/ digitalhealth-TB-agenda/en/

23 Mirsaeidi M, Farshidpour M, Banks-Tripp D, et al. Video directly observed therapy for treatment of tuberculosis is patient-oriented and cost-effective. Eur Respir J 2015; 46: 871-874.

24 Krueger K, Ruby D, Cooley P, et al. Videophone utilization as an alternative to directly observed therapy for tuberculosis. Int J Tuberc Lung Dis 2010; 14: 779-781.

25 International Telecommunication Union. ICT-Eye. Brazil Country Profile 2015. Available from: www.itu.int/icteye. Date last accessed: August 29, 2018.

26 Broomhead S, Mars M. Retrospective return on investment analysis of an electronic treatment adherence device piloted in the Northern Cape Province. Telemed J E Health 2012; 18: 24-31.

27 Sabin LL, DeSilva MB, Hamer DH, et al. Using electronic drug monitor feedback to improve adherence to antiretroviral therapy among HIV-positive patients in China. AIDS Behav 2010; 14: 580-589.

28 Sabin LL, DeSilva MB, Gill CJ, et al. Improving adherence to antiretroviral therapy with triggered real-time text message reminders: the China Adherence through Technology Study. J Acquir Immune Defic Syndr 2015; 69: 551-559.

29 Cross A, Rodrigues R, D’Souza G, et al. 99DOTS: Using Mobile Phones to Monitor Adherence to Tuberculosis Medications. Washington, Global mHealth Forum, 2014. Available from: www.microsoft.com/en-us/research/ publication/99dots-using-mobile-phones-monitor-adherence-tuberculosis-medications/

30 Oberoi S, Gupta VK, Chaudhary N, et al. 99 DOTS. Int J Contemp Med Res 2016; 3: 2760-2762.

31 Chuck C, Robinson E, Macaraig M, et al. Enhancing management of tuberculosis treatment with video directly observed therapy in New York City. Int J Tuberc Lung Dis 2016; 20: 588-593.

32 van der Kop ML, Memetovic J, Smillie K, et al. Use of the WelTel mobile health intervention at a tuberculosis clinic in British Columbia: a pilot study. J Mobile Technol Med 2013; 2: 7-14.

33 Liu X, Lewis JJ, Zhang $\mathrm{H}$, et al. Effectiveness of electronic reminders to improve medication adherence in tuberculosis patients: a cluster-randomised trial. PLoS Med 2015; 12: e1001876.

34 Lester RT, Ritvo P, Mills EJ, et al. Effects of a mobile phone short message service on antiretroviral treatment adherence in Kenya (WelTel Kenya1): a randomised trial. Lancet 2010; 376: 1838-1845.

35 Mohammed S, Glennerster R, Khan AJ. Impact of a daily SMS medication reminder system on tuberculosis treatment outcomes: a randomized controlled trial. PLoS One 2016; 11: e0162944.

36 Salomon JA, Haagsma JA, Davis A, et al. Disability weights for the Global Burden of Disease 2013 study. Lancet Glob Health 2015; 3: e712-e723.

37 Johnston JC, van der Kop ML, Smillie K, et al. The effect of text messaging on latent tuberculosis treatment adherence: a randomised controlled trial. Eur Respir J 2018; 51: 1701488

38 PitchWink. www.pitchwink.com Date last accessed: August 29, 2018

39 Hwang B, Coleman J, Lester R. WelTel Kenya: Business case for using mobile phones as a cost-effective health intervention to provide care and support HIV/AIDS patients. Presented at: Third International Conference for Improving Use of Medicines: Informed Strategies, Effective Policies, Lasting Solutions. Antalya, 2011. Abstract 889. Available from: http://apps.who.int/medicinedocs/en/d/Js21782en/

40 Oliveira GP, Torrens AW, Bartholomay P, et al. Tuberculosis in Brazil: last ten years analysis - 2001-2010. Braz Infect Dis 2013; 17: 218-233.

41 Programa Nacional de Controle da Tuberculose. Manual de Recomendações para o Controle da Tuberculose no Brasil [Guidelines for Tuberculosis Control in Brazil]. Brasilia, Ministério da Saúde, 2011. http://bvsms.saude.gov. br/bvs/publicacoes/manual_recomendacoes_controle_tuberculose_brasil.pdf

42 Pooran A, Pieterson E, Davids $\mathrm{M}$, et al. What is the cost of diagnosis and management of drug resistant tuberculosis in South Africa? PLoS One 2013; 8: e54587. 
43 Departamentode Informática do Sistema Único de Saúde (DATASUS). Hospitalisation costs. 2016. Available from: www2.datasus.gov.br Date last accessed: July 5, 2018.

44 Castro KG, Marks SM, Chen MP, et al. Estimating tuberculosis cases and their economic costs averted in the United States over the past two decades. Int J Tuberc Lung Dis 2016; 20: 926-933.

45 Free C, Phillips G, Galli L, et al. The effectiveness of mobile-health technology-based health behaviour change or disease management interventions for health care consumers: a systematic review. PLoS Med 2013; 10: e1001362.

46 Gurol-Urganci I, de Jongh T, Vodopivec-Jamsek V, et al. Mobile phone messaging reminders for attendance at healthcare appointments. Cochrane Database Syst Rev 2013; 12: CD007458.

47 Gagnon MP, Ngangue P, Payne-Gagnon J, et al. m-Health adoption by healthcare professionals: a systematic review. J Am Med Inform Assoc 2016; 23: 212-220.

48 Muench F. The promises and pitfalls of digital technology in its application to alcohol treatment. Alcohol Res 2014; 36: 131-142.

49 Nguyen TA, Pham MT, Nguyen TL, et al. Video directly observed therapy to support adherence with treatment for tuberculosis in Vietnam: a prospective cohort study. Int J Infect Dis 2017; 65: 85-89.

50 Christensen H, Griffiths KM, Farrer L. Adherence in internet interventions for anxiety and depression. J Med Internet Res 2009; 11: e13.

51 Matthew-Maich N, Harris L, Ploeg J, et al. Designing, implementing, and evaluating mobile health technologies for managing chronic conditions in older adults: a scoping review. JMIR Mhealth Uhealth 2016; 4: e29.

52 Sterling TR, Villarino ME, Borisov AS, et al. Three months of rifapentine and isoniazid for latent tuberculosis infection. N Engl J Med 2011; 365: 2155-2166.

53 Belknap R, Holland D, Feng PJ, et al. Self-administered versus directly observed once-weekly isoniazid and rifapentine treatment of latent tuberculosis infection: a randomized trial. Ann Intern Med 2017; 167: 689-697. 